\title{
Large deformations of 1D microstructured systems modeled as generalized Timoshenko beams
}

\author{
A. Battista - A. Della Corte - F. \\ dell'Isola · P. Seppecher
}

Received: date / Accepted: date

\begin{abstract}
In the present paper we study a natural nonlinear generalization of Timoshenko beam model and show that it can describe the homogenized deformation energy of a 1D continuum with a simple microstructure. We prove the well-posedness of the corresponding variational problem in case of a generic end load, discuss some regularity issues and evaluate the critical load. Moreover, we generalize the model so as to include an additional rotational spring in the microstructure. Finally, some numerical simulations are presented and discussed.
\end{abstract}

Keywords Nonlinear elasticity · Generalized Timoshenko beam · Microstructured beam · Non-convex variational problems

\author{
A. Battista \\ Uiversité de La Rochelle, La Rochelle, France \\ M\&MoCS, Research Center, University of L'Aquila, L'Aquila, Italy \\ E-mail: antoniobattista1986@gmail.com \\ A. Della Corte \\ University La Sapienza, Rome, Italy \\ M\&MoCS, Research Center, University of L'Aquila, L'Aquila, Italy \\ E-mail: alessandro.dellacorte.memocs@gmail.com \\ F. dell'Isola \\ University La Sapienza, Rome, Italy \\ M\&MoCS, Research Center, University of L'Aquila, L'Aquila, Italy \\ E-mail: francesco.dellisola@uniroma1.it \\ P. Seppecher \\ IMATH-Université de Toulon, Toulon, France \\ M\&MoCS, Research Center, University of L'Aquila, L'Aquila, Italy \\ E-mail: seppecher@imath.fr
}




\section{Introduction}

Your text comes here. Separate text sections with The main aim of this paper is to study the problem of large deformations of a microstructured beam, which can be described at the macroscopic level by means of a generalized Timoshenko model. We focus on the case of a beam clamped at one endpoint and submitted to a concentrated load at the other endpoint.

Timoshenko proposed the first beam model going beyond the classical Euler model. The latter was formulated around mid-18th century [1], and scientists of the caliber of Daniel and Jakob Bernoulli, as well as Lagrange, gave important contributions to its theory [2-4]. The model has been rigorously deduced from $3 \mathrm{D}$ elasticity $[5,6]$ and it is widely applied in many problems of structural mechanics (a useful reference book is [7]).

Let $\mathcal{E}$ be the affine euclidean plane and $\left\{\boldsymbol{e}_{1}, \boldsymbol{e}_{2}\right\}$ an orthonormal basis. The deformation energy of an inextensible Euler beam can be written as:

$$
\int_{0}^{L} \frac{k_{b}}{2} \chi^{\prime \prime}(s) \cdot \chi^{\prime \prime}(s) d s=\int_{0}^{L} \frac{k_{b}}{2} \eta^{2}(s) d s
$$

under the inextensibility constraint

$$
\left\|\chi^{\prime}(s)\right\|=1 \quad \forall s \in[0, L]
$$

where $L$ is the length of the beam (assumed straight and parallel to $\boldsymbol{e}_{1}$ in the reference configuration), $\chi$ is the placement, i.e. a bijective continuous function mapping material points of the beam (labeled with $s \in[0, L])$ into $\mathcal{E}$, and $k_{b}$ is a material parameter accounting for the bending stiffness. In the present paper, $(.)^{\prime}$ denotes denotes differentiation with respect to $s$. In particular, $\eta(s)=\left\|\chi^{\prime \prime}(s)\right\|$ is the absolute value of the curvature of the current shape of the beam. Note that, although the deformation energy (1) is a quadratic form, the inextensibility constraint (2) is not convex.

Since in most of applications to structural mechanics deflections are small when compared to the length of the beams, it is very usual to consider, instead of the previous one, the approximated linearized model. In this case, constraint (2) becomes $\chi_{1}=s$ and the deformation energy reads:

$$
\int_{0}^{L} \frac{k_{b}}{2}\left(\chi_{2}^{\prime \prime}\right)^{2} d s
$$

Static problems for the commonly employed linearized model lead to fourthorder linear ODEs, while the nonlinear model originarily formulated by Euler leads to semilinear fourth-order ODEs.

The nonlinear Euler model can be reformulated in terms of the variable $\theta$ satisfying

$$
\chi^{\prime}=\cos \theta \boldsymbol{e}_{1}+\sin \theta \boldsymbol{e}_{2}
$$


The energy becomes:

$$
\int_{0}^{L} \frac{k_{b}}{2} \theta^{\prime 2}(s) d s
$$

The beam is said to be clamped at the left extremum if $\chi(0)=\mathbf{0}$ and $\chi^{\prime}(0)=$ $\mathbf{e}_{1}$. An external potential $\boldsymbol{b}(s) \cdot \chi(s)$, where $\boldsymbol{b}(s)$ is a distributed load, can also be written in terms of $\theta$ by performing an integration by parts. The total energy of the system becomes:

$$
E(\theta)=\int_{0}^{L}\left[\frac{k_{b}}{2} \theta^{\prime 2}(s)-B_{1} \cos \theta(s)-B_{2}(s) \sin \theta(s)\right] d s
$$

where $\boldsymbol{B}=B_{1} \mathbf{e}_{1}+B_{2} \mathbf{e}_{2}$ denotes the primitive of $\boldsymbol{b}$ verifying $\boldsymbol{B}(L)=0$. It is natural to search for minimizers of the energy (6) in the set of functions belonging to $H^{1}:=W^{1,2}$ verifying $\theta(0)=0$ in the sense of traces. We remark that the angle $\theta(s)$ is uniquely determined by equation (4) if one takes into account the clamp condition $\theta(0)=0$ and the fact that $H^{1}$ functions have continuous representatives. This reformulation automatically takes into account the inextensibility constraint and the non-convexity of the minimization problem appears clearly.

Timoshenko beam model, was introduced to describe in a more precise way the shear deformation of the beam (the problem is addressed in an original way in [8]). It was developed much later, in the early 1920s [9], and motivated by several applications. Specifically, for the static case, it was needed for describing beams that were not so slender, in which case shear deformation is no more negligible. This is also the case with sandwich composite beams ([10]). Timoshenko beam model is still an important tool for current research: the possibility of very precisely manufacturing the inner architecture of beams (e.g. by means of 3D-printing) makes it now possible to produce slender objects which display a richer behavior than what can be captured by Euler beam model (see e.g. [11-13] for interesting examples, [14-16] for cases in which dynamical/instability problems are addressed and [17-20] for an approach using asymptotic justification; a review of complex structures employing fibers that can be modeled as generalized beams is [21]).

The original model from Timoshenko was established in a linear framework. The deformation energy of an inextensible linear Timoshenko beam reads:

$$
\int_{0}^{L}\left[\frac{K_{1}}{2}\left(\phi^{\prime}(s)\right)^{2}+\frac{K_{2}}{2}\left(\phi(s)-u_{2}^{\prime}(s)\right)^{2}\right] d s
$$

where $\phi$ is an independent kinematic descriptor and $K_{1}$ and $K_{2}$ are material parameters. In the original interpretation of the model, $\phi$ was thought to measure the angle between the cross section of the beam and a reference axis, and therefore the model can be seen as a particular case of a Cosserat continuum (see e.g. [22-25]).

Clearly there are infinitely many nonlinear generalizations of the previous linearized energy model. A possible generalization can be obtained by replacing, similarly to the Euler case, the term $u_{2}^{\prime}$ by the angle $\theta$ defined as above: 


$$
\int_{0}^{L}\left[\frac{K_{1}}{2}\left(\phi^{\prime}(s)\right)^{2}+\frac{K_{2}}{2}(\phi(s)-\theta(s))^{2}\right] d s
$$

This energy model is a natural generalization of the linear one in the following sense: it admits the nonlinear Euler beam model as a limit case exactly in the same sense in which the linear Timoshenko model admits the linearized Euler model, i.e. the corresponding Euler-Lagrange equation tends to the equation describing the nonlinear Euler beam when the shear stiffness parameter goes to infinity. We will obtain this energy model with a formal homogenization starting from a microstructure consisting of articulated parallelograms and rotational springs.

The paper is organized as follows: in section 2 various kinds of microstructures are considered and in particular a novel one is introduced which leads to the deformation energy (8); moreover, an additional rotational spring is also considered to introduce a regularizing term which further generalizes the model. In section 3 the well-posedness of the variational problem given by the equilibria of a clamped beam under end load is addressed, some regularity issues are discussed and the critical load is evaluated. In section 4 numerical simulations are presented and discussed. In section 5 some conclusions are provided as well as possible directions for future researches.

\section{Microscopic interpretation of a Timoshenko beam}

\subsection{The linearized case}

A traditional way of introducing Timoshenko model in structural mechanics courses is to provide a microscopical interpretation of it by means of a discrete system of rotational and extensional springs. Nowadays, the possibility of accurately 3D-printing the microstructure makes these discretizations more than academic examples, but rather possibilities to concretely implement mechanical systems with certain desired properties.

Let us consider the system illustrated in Fig. 1. The length of the deformed extensional springs connecting the points $\boldsymbol{t}_{i}^{+}$and $\boldsymbol{t}_{i+1}^{+}$can be computed as:

$$
\begin{aligned}
& \left\|\boldsymbol{t}_{i+1}^{+}-\boldsymbol{t}_{i}^{+}\right\|^{2}= \\
& =\varepsilon^{2}\left\{1+2 h\left[\sin \left(\theta_{i}-\phi_{i+1}\right)+\sin \left(\phi_{i}-\theta_{i}\right)\right]+2 h^{2}\left[1-\cos \left(\phi_{i+1}-\phi_{i}\right)\right]\right\}
\end{aligned}
$$

Now if we assume that the quantities $\theta$ and $\phi$, which measure the deformation of the system, remain small, we can write the following approximate form:

$$
\left\|\boldsymbol{t}_{i+1}^{+}-\boldsymbol{t}_{i}^{+}\right\| \approx \varepsilon\left[1+h\left(\phi_{i}-\phi_{i-1}\right)\right]
$$

Let us assume that all the extensional springs are linear (with elastic constant $\left.k_{1} /\left(2 h^{2}\right)\right)$. Performing a formal passage to the limit we have: 


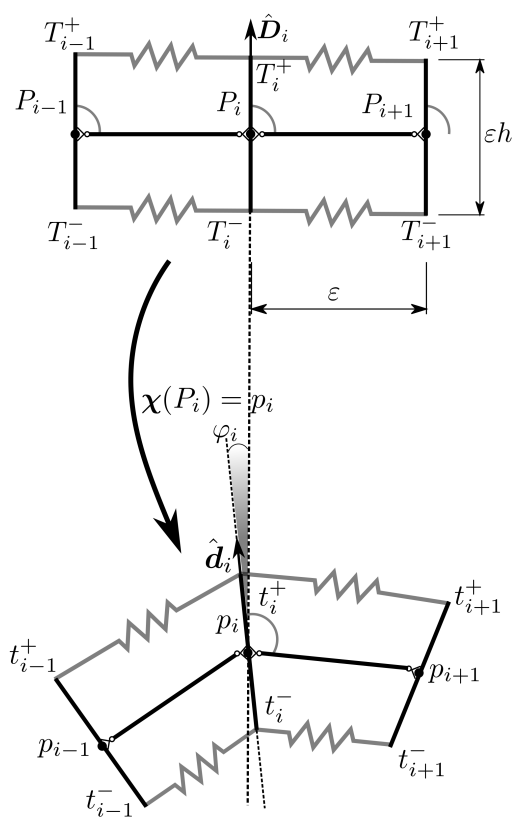

Fig. 1 The bar-spring microstructure usually introduced to get the linear Timoshenko model valid for small deformations (top: reference configuration; bottom: actual configuration). For finite deformations, a heuristic homogenization for this microstructure, leads to the energy (14).

$$
\frac{1}{2} \frac{k_{1}}{2 h^{2}}\left(\left\|\boldsymbol{t}_{i+1}^{+}-\boldsymbol{t}_{i}^{+}\right\|-\varepsilon\right)^{2} \approx \frac{1}{2} \frac{k_{1}}{2} \phi^{\prime 2} \varepsilon^{4}
$$

An analogous contribution in the energy will come from the spring connecting $\boldsymbol{t}_{i}^{-}$and $\boldsymbol{t}_{i+1}^{-}$. The potential energy of the rotational spring connecting $\boldsymbol{t}_{i}^{-} \boldsymbol{t}_{i}^{+}$ and $\boldsymbol{p}_{i} \boldsymbol{p}_{i+1}$ is assumed to be:

$$
\frac{1}{2} k_{2}\left(\phi_{i}-\theta_{i}\right)^{2}
$$

where $k_{2}$ is the elastic constant of the spring.

The deformation energy of a microstructured system composed of $N$ elementary cells like the two represented in Fig. 1, in the linearized case, can be therefore written as:

$$
\sum_{i=1}^{i=N}\left[\frac{1}{2} k_{1} \phi^{\prime 2} \varepsilon^{4}+\frac{1}{2} k_{2}\left(\phi_{i}-\theta_{i}\right)^{2}\right] .
$$

In the limit for $\varepsilon \rightarrow 0$ the stiffnesses have to be suitably rescaled: $k_{1}=K_{1} \varepsilon^{-4}$ and $k_{2}=K_{2}$. Recalling that, in our hypotheses, $\theta$ is small, we can approximate it by means of $u_{2}^{\prime}$, obtaining for the homogenized energy formula (7). 


\subsection{The nonlinear case}

If one does not want to assume that the angles $\phi$ and $\theta$ remain small, the energy of the previous discrete system takes a more complicated form.

Indeed, the continuous form of (9) is:

$$
\frac{1}{2} \frac{k_{1}}{2 h^{2}}\left(\left\|\boldsymbol{t}_{i+1}^{+}-\boldsymbol{t}_{i}^{+}\right\|-\varepsilon\right)^{2} \approx \frac{1}{2} k_{1} \phi^{\prime 2} \cos ^{2}(\theta-\phi) \varepsilon^{4}
$$

and the homogenized energy, with the same scaling as before, becomes

$$
\int_{0}^{L}\left[\frac{1}{2} K_{1}\left(\phi^{\prime}\right)^{2} \cos ^{2}(\theta-\phi)+\frac{1}{2} K_{2}(\phi-\theta)^{2}\right]
$$

As we can see, the last expression does not coincide with the form of Timoshenko deformation energy given in formula (8) because of the the factor $\cos ^{2}(\theta-\phi)$. While the microstructure relative to Fig.1 has an intrinsic physical interest, the homogenized energy (14) has a more complex structure than (8) and we consider more suitable to attack the simplest problem first. Indeed, we can recover the energy model (8) by means of a different microstructure (see Fig. 2).

We start by considering an articulated parallelogram in which all the sides are rigid bars, and we add another rigid bar connecting the middle points of two sides. Then we organize them in series (see Fig 2). The points $R_{i-1}^{+}$and $L_{i}^{+}$, $R_{i-1}^{-}$and $L_{i}^{-}$as well as $P_{i}^{r}$ and $P_{i-1}^{\ell}$ coincide in the reference configuration, so that the two bars $\overrightarrow{R_{i-1}^{+} R_{i-1}^{-}}$and $\overrightarrow{L_{i}^{+} L_{i}^{-}}$are superposed. We describe the mechanical interaction between the bars by means of rotational springs. We introduce two rotational springs:

1. one between the directions of $\overrightarrow{R_{i} P_{i}}$ and $\overrightarrow{P_{i+1} P_{i}}$

2. one between the directions of $\overrightarrow{R_{i} P_{i}}$ and $\overrightarrow{L_{i+1} P_{i+1}}$

Assuming that the rotational springs are linear in the angle, the deformation energy takes the form:

$$
\sum_{i=1}^{N}\left[\frac{k_{1}}{2}\left(\phi_{i}-\phi_{i-1}\right)^{2}+\frac{k_{2}}{2}\left(\phi_{i}-\theta_{i}\right)^{2}\right]
$$

and setting $K_{1}:=k_{1} \varepsilon^{4}, K_{2}:=k_{2}$, formula (8) is recovered with a formal homogenization procedure. In the following, we will call the energy model (8) Nonlinear Timoshenko model.

Finally, we can introduce an additional rotational spring, i.e.:

3. between the directions of $\overrightarrow{P_{i} P_{i-1}}$ and $\overrightarrow{P_{i+1} P_{i}}$

In this case (assuming that the new rotational spring has stiffness $k_{3}$ and setting $K_{3}:=k_{3} \varepsilon^{4}$ ), an analogous formal homogenization procedure leads to the following energy functional: 

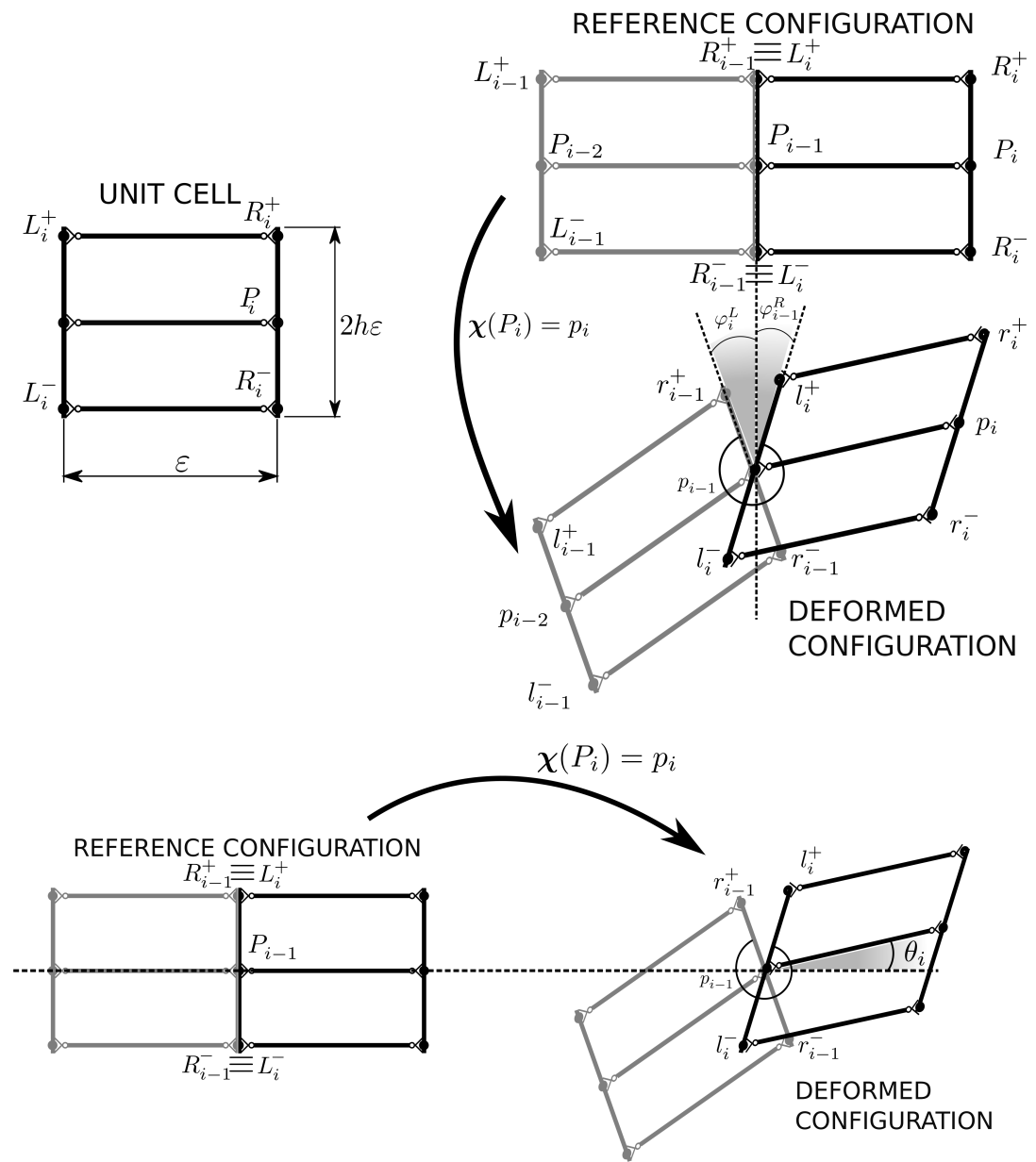

Fig. 2 Another possible micro-structure for shear deformable beams producing (after linearization) the Timoshenko beam model. On top: the unit cell and a graphical representation of the variation of the cross-section angle $\phi$. At the bottom: graphical representation of the variation of angle $\theta$ formed by the tangent to the deformed shape). A heuristic homogenization of this micro structure leads do the energy (8).

$$
\int_{0}^{L}\left[\frac{K_{1}}{2}\left(\phi^{\prime}\right)^{2}+\frac{K_{2}}{2}(\phi-\theta)^{2}+\frac{K_{3}}{2}\left(\theta^{\prime}\right)^{2}\right] d s
$$

In the following, we will call the energy model (16) Regularized Timoshenko model. 


\section{Properties of the minimization problems}

\subsection{Nonlinear Timoshenko model}

Let us consider the deformation energy (8) in the model case in which a concentrated load $\boldsymbol{F}=F_{1} \boldsymbol{e}_{1}+F_{2} \boldsymbol{e}_{2}$ is applied at the free endpoint. We are led to the following variational problem:

$$
\begin{gathered}
\min \left\{E(\phi, \theta):=\int_{0}^{L}\left[\frac{K_{1}}{2} \phi^{2}+\frac{K_{2}}{2}(\phi-\theta)^{2}+F_{1} \cos \theta+F_{2} \sin \theta\right] d s\right. \\
\left.\theta \in L^{2}[0, L], \quad \phi \in H^{1}[0, L], \quad \phi(0)=0\right\}
\end{gathered}
$$

\subsubsection{Well-posedness}

We prove in this section that problem (17) is well posed. Let us first rewrite the problem in a non dimensional form. A change of length and energy units leads to

$$
\begin{gathered}
\min \left\{\tilde{E}(\phi, \theta):=\int_{0}^{1}\left[\frac{1}{2} \phi^{2}+\tilde{K}_{2}\left(\frac{1}{2}(\phi-\theta)^{2}+\tilde{F} \cos (\theta-\gamma)\right)\right] d s\right. \\
\left.\theta \in L^{2}[0,1], \quad \phi \in H^{1}[0,1], \quad \phi(0)=0\right\}
\end{gathered}
$$

where $\tilde{K}_{2}:=\frac{K_{2} L^{2}}{K_{1}}$ and $(\tilde{F}, \gamma) \in[0,+\infty) \times[0,2 \pi[$ are defined by:

$$
\tilde{F}(\cos (\gamma), \sin (\gamma))=K_{2}^{-1}\left(F_{1}, F_{2}\right) .
$$

Proposition 1 Problem (18) admits a solution.

Proof Let $G(\theta):=\frac{\theta^{2}}{2}+\tilde{F} \cos (\theta-\gamma)$ and $G^{*}(z)$ its convex conjugate, defined by $G^{*}(z):=\max _{\theta}[z \theta-G(\theta)]$. Since $G$ is continuous and coercive, there exists $\bar{\theta}$ solving the max problem and $\bar{\theta}(z)$ belongs to the subdifferential $\partial G^{*}(z)$. We note that $G^{*}(z)$ is not differentiable in correspondence of intervals in which $G$ does not coincide with its lower convex envelop.

Hence:

$$
\begin{aligned}
& \inf _{\theta, \phi} \tilde{E}(\theta, \phi)=\inf _{\theta, \phi} \int_{0}^{1}\left[\frac{1}{2} \phi^{\prime 2}+\tilde{K}_{2}\left(\frac{1}{2} \phi^{2}+(-\phi \theta+G(\theta))\right)\right] d s= \\
& \inf _{\phi} \int_{0}^{1}\left[\frac{1}{2} \phi^{\prime 2}+\tilde{K}_{2}\left(\frac{1}{2} \phi^{2}+\inf _{\theta}(-\phi \theta+G(\theta))\right)\right] d s= \\
& \inf _{\phi} \int_{0}^{1}\left[\frac{1}{2} \phi^{\prime 2}+\tilde{K}_{2}\left(\frac{1}{2} \phi^{2}-\sup _{\theta}(\phi \theta-G(\theta))\right)\right] d s=\inf _{\phi} \mathcal{A}(\phi)
\end{aligned}
$$


where

with

$$
\mathcal{A}(\phi):=\int_{0}^{1}\left(\frac{1}{2} \phi^{\prime 2}+\tilde{K}_{2} h(\phi)\right) d s
$$

$$
h(\phi):=\frac{1}{2} \phi^{2}-G^{*}(\phi) .
$$

Let $\left(\theta_{n}, \phi_{n}\right)$ be a minimizing sequence. Then $\tilde{E}\left(\theta_{n}, \phi_{n}\right)$ is a bounded sequence and thus $\int_{0}^{1} \frac{1}{2}\left(\phi_{n}^{\prime}\right)^{2}$ is also bounded and $\phi_{n}(0)=0$. Thus $\phi_{n}$ is bounded in $H^{1}$ : there exists a subsequence (still denoted $\phi_{n}$ ) weakly converging in $H^{1}$ to some function $\bar{\phi}$. Remind that in the one-dimensional case $H^{1}$-weak convergence implies uniform convergence and note also that $G^{*}$ is convex, and therefore it is locally Lipschitz (see Theorem 3.7.3 in [26]). Thus $\int_{0}^{1}\left[\phi_{n}^{2} / 2-G^{*}\left(\phi_{n}\right)\right] d s$ converges to $\int_{0}^{1}\left[\bar{\phi}^{2} / 2-G^{*}(\bar{\phi})\right] d s$. On the other hand, as convexity and weak convergence imply $\liminf _{n} \int_{0}^{1} \phi_{n}^{\prime 2} d s \geq \int_{0}^{1} \bar{\phi}^{\prime 2} d s$, the functional $\mathcal{A}$ is $H^{1}$-lower semicontinuous and $\bar{\phi}$ is a global minimizer for $\mathcal{A}$. Finally let us remark that the constraint $\phi_{n}(0)=0$ passes to the uniform limit: $\bar{\phi}(0)=0$.

The function $\bar{\theta}(s)$, which solves:

$$
\max _{\theta}[\bar{\phi}(s) \theta-G(\theta)]
$$

is uniquely defined everywhere on $[0,1]$, by the equation

$$
\frac{d G^{* *}(\theta)}{d \theta}=\bar{\phi}(s)
$$

or equivalently by

$$
\theta=\left.\frac{d G^{*}(z)}{d z}\right|_{z=\bar{\phi}(s)}
$$

except at the points $s$ such that $G^{*}(\bar{\phi}(s))$ is not differentiable. As the convex conjugate of $G$ is piecewise $C^{\infty 1}, \theta$ is also $C^{\infty}$ on the intervals in which it is uniquely determined.

Since $\bar{\phi} \in H^{1}(0,1)$ is continuous in $[0,1]$, it attains a maximum $\phi_{M}$ and a minimum $\phi_{m}$, and therefore we are interested in the differentiability of $G^{*}$ only in the interval $\left[\phi_{m}, \phi_{M}\right]$. The set $\left\{\phi_{1}, \ldots, \phi_{k}\right\}$ of the points which belong to $\left[\phi_{m}, \phi_{M}\right]$ and where $G^{*}$ is not differentiable is finite. Let us now prove by contradiction that $\bar{\phi}$ takes the values $\phi_{1}, \ldots, \phi_{k}$ only on a subset of $[0,1]$ of measure zero. Otherwise there would exist an integer $i \in\{1, \ldots, k\}$ such that the measure of $\bar{\phi}^{-1}\left(\phi_{i}\right)$ is positive. Adapting known monotonicity results for autonomous variational problems (see e.g. Theorem 3.1 in [28]), we know that $\bar{\phi}$ is monotonic ${ }^{2}$ and thus that $\bar{\phi}^{-1}\left(\phi_{i}\right)$ is an interval. The following lemma states that for this reason $G^{*}$ is differentiable at $\phi_{i}$, which contradicts the definition of $\phi_{i}$. In conclusion $\bar{\theta}$ is uniquely determined almost everywhere, it is bounded because $G^{*}$ is Lipschitz on $\left[\phi_{m}, \phi_{M}\right]$ and therefore it belongs to $L^{2}[0,1]$.

\footnotetext{
1 This, for instance, is a (trivial) particular case of Theorem 26.1 in [27]

2 The cited theorem is stated for a problem with values prescribed at both ends, but is true also in the present case. One just has to apply it to a problem having $\phi(1)=\bar{\phi}(1)$.
} 


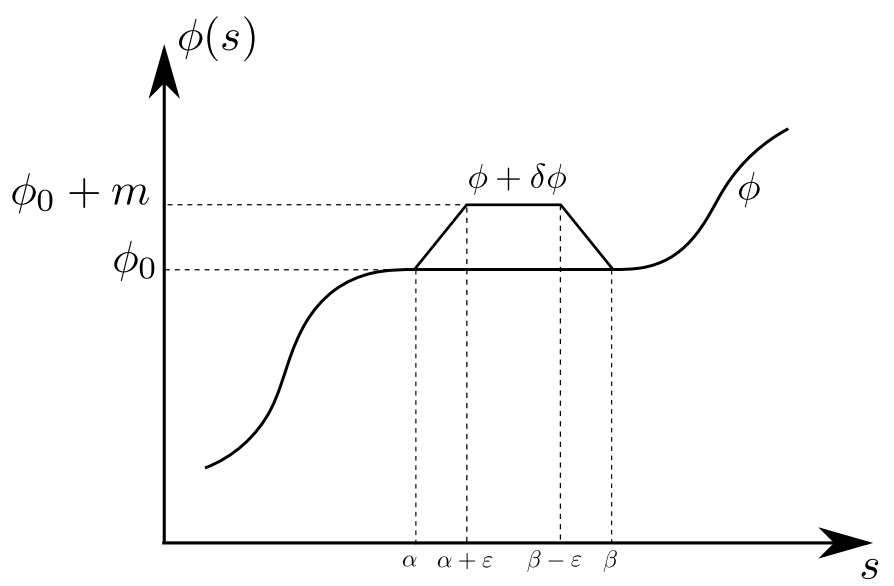

Fig. 3 Representation of $\phi$ and of the considered variation $\delta \phi$

Lemma 1 Let $\bar{\phi}$ be a minimizer of $\mathcal{A}$. Suppose that there exists an interval having positive measure $[\alpha, \beta] \subset[0,1]$ in which $\bar{\phi}(s)=\phi_{0}$ is constant. Then $G^{*}$ is differentiable at $\phi_{0}$, and $\frac{\partial G^{*}}{\partial \phi}\left(\phi_{0}\right)=\phi_{0}$.

Proof We first observe that since $G^{*}$ is convex, it admits left and right derivatives and therefore for every $\phi$ there exist both the right and left derivatives $\frac{\partial h}{\partial \phi}^{+}(\phi)$ and $\frac{\partial h}{\partial \phi}^{-}(\phi)$.

Now let $\varepsilon$ be such that $0<\varepsilon<\frac{\beta-\alpha}{2}$ and let $m$ and $c>0$ be real numbers such that $\varepsilon=\sqrt{|m| / 2 c}<(\beta-\alpha) / 2$.

Let us consider a variation $\delta \phi$ defined as (see Fig. 3):

$$
\left\{\begin{array}{l}
\delta \phi(s):=0 \quad \text { for } \quad s \in[0, \alpha) \cup(\beta, 1] \\
\delta \phi(s):=\frac{m}{\varepsilon} s-\frac{m}{\varepsilon} \alpha \text { for } s \in[\alpha, \alpha+\varepsilon] \\
\delta \phi(s):=-\frac{m}{\varepsilon} s+\frac{m}{\varepsilon} \beta \text { for } s \in[\beta-\varepsilon, \beta] \\
\delta \phi(s):=m \text { for } s \in(\alpha+\varepsilon, \beta-\varepsilon)
\end{array}\right.
$$

Clearly $[\bar{\phi}+\delta \phi] \in H^{1}$ and $[\bar{\phi}+\delta \phi](0)=0$. Let us set $\Delta h(\delta \phi):=h(\bar{\phi}+\delta \phi)-$ $h(\bar{\phi})$. We have:

$$
\begin{aligned}
0 & \leq \Delta \mathcal{A}:=\mathcal{A}(\bar{\phi}+\delta \phi)-\mathcal{A}(\bar{\phi})= \\
& =\int_{\alpha}^{\beta} \frac{1}{2} \delta \phi^{\prime 2} d s+\tilde{K}_{2}\left(\int_{\alpha}^{\alpha+\varepsilon} \Delta h(\delta \phi) d s+\int_{\alpha+\varepsilon}^{\beta-\varepsilon} \Delta h(\delta \phi) d s+\int_{\beta-\varepsilon}^{\beta} \Delta h(\delta \phi) d s\right)
\end{aligned}
$$


Using that $h$ is $c$-Lipschitz on the compact set $\left[\phi_{m}-|m|, \phi_{M}+|m|\right]$, one gets:

$$
0 \leq \frac{m^{2}}{\varepsilon}+\tilde{K}_{2}\left((\beta-\alpha-2 \varepsilon)\left(h\left(\phi_{0}+m\right)-h\left(\phi_{0}\right)\right)+2 c \varepsilon|m|\right)
$$

The last inequality implies:

$$
h\left(\phi_{0}+m\right)-h\left(\phi_{0}\right) \geq-\frac{2 \sqrt{2 c}}{\tilde{K}_{2}(\beta-\alpha-2 \varepsilon)}|m|^{3 / 2}
$$

In terms of $G^{*}$, the inequality $(23)$ can be written as:

$$
G^{*}\left(\phi_{0}+m\right)-G^{*}\left(\phi_{0}\right) \leq \frac{m^{2}}{2}+\phi_{0} m+\frac{2 \sqrt{2 c}}{\tilde{K}_{2}(\beta-\alpha-2 \varepsilon)}|m|^{3 / 2}
$$

The left hand side of (24) is a convex function of $m$ while the right hand side is a $C^{1}$ function of $m$. Both sides coincide when $m=0$. It is easily seen that a convex function bounded from above by a $C^{1}$ function is differentiable at any coinciding point. Therefore $G^{*}$ is differentiable at $\phi=\phi_{0}$.

Dividing by $m$ both sides of (24) and letting $m \rightarrow 0^{+}$or $m \rightarrow 0^{-}$, we obtain:

$$
\left(\frac{\partial G^{*}}{\partial \phi}\right)^{+}\left(\phi_{0}\right) \leq \phi_{0} \quad \text { and } \quad\left(\frac{\partial G^{*}}{\partial \phi}\right)^{-}\left(\phi_{0}\right) \geq \phi_{0} .
$$

As a consequence

$$
\frac{\partial G^{*}}{\partial \phi}\left(\phi_{0}\right)=\phi_{0}
$$

\subsubsection{Regularity}

We address now the properties of the function $h$. In order to establish its regularity properties, we first have to provide some preliminary results.

Let us first remark that $h$ is a $2 \pi$-periodic function. Indeed, for any $z \in \mathbb{R}$, let us denote $[z]$ the integer part of $\frac{z-\gamma}{2 \pi}$, and let us set $\tilde{z}:=z-2 \pi[z]$. We can write

$$
\begin{aligned}
G^{*}(z) & =\max _{\theta}\left[z \theta-\frac{\theta^{2}}{2}-F \cos (\theta-\gamma)\right] \\
& =\max _{\theta}\left[2 \pi^{2}[z]^{2}+2 \pi[z] \tilde{z}+\tilde{z}(\theta-2 \pi[z])-\frac{1}{2}(\theta-2 \pi[z])^{2}-F \cos (\theta-\gamma)\right] \\
& =\max _{\alpha}\left[2 \pi^{2}[z]^{2}+2 \pi[z] \tilde{z}+\tilde{z} \alpha-\frac{1}{2} \alpha^{2}-F \cos (\alpha-\gamma)\right] \\
& =2 \pi^{2}[z]^{2}+2 \pi[z] \tilde{z}+G^{*}(\tilde{z}) .
\end{aligned}
$$

where we introduced the variable $\alpha:=\theta-2 \pi[z]$. Hence:

$$
\begin{aligned}
h(z) & =\frac{z^{2}}{2}-G^{*}(z) \\
& =\frac{\tilde{z}^{2}}{2}+2 \pi^{2}[z]^{2}+2 \pi[z] \tilde{z}-\left(2 \pi^{2}[z]^{2}+2 \pi[z] \tilde{z}+G^{*}(\tilde{z})\right) \\
& =\frac{\tilde{z}^{2}}{2}-G^{*}(\tilde{z})=h(\tilde{z}) .
\end{aligned}
$$


We address now the dependence of $h$ on $\gamma$. For that aim let us define

$$
G_{\gamma}(\theta):=\frac{\theta^{2}}{2}+\tilde{F} \cos (\theta-\gamma)
$$

and

$$
h_{\gamma}(z)=\frac{z^{2}}{2}-G_{\gamma}^{*}(z) .
$$

We have

$$
\begin{aligned}
h_{\gamma}(z) & =\frac{z^{2}}{2}-\max _{\theta}\left[\theta z-\frac{\theta^{2}}{2}-\tilde{F} \cos (\theta-\gamma)\right] \\
& =\frac{z^{2}}{2}-\max _{\alpha}\left[(\alpha+\gamma) z-\frac{1}{2}(\alpha+\gamma)^{2}-\tilde{F} \cos \alpha\right] \\
& =\frac{z^{2}}{2}-\gamma z+\frac{\gamma^{2}}{2}-\max _{\alpha}\left[(z-\gamma) \alpha-\frac{\alpha^{2}}{2}-\tilde{F} \cos \alpha\right] \\
& =\frac{(z-\gamma)^{2}}{2}-G_{0}^{*}(z-\gamma)=h_{0}(z-\gamma) .
\end{aligned}
$$

Therefore the regularity properties of $h_{\gamma}$ result from those of $h_{0}$. Remarking that $h_{0}$ like $G_{0}^{*}$ is clearly even, we are reduced to study $h_{0}$ on the interval $[0, \pi]$. We can now address the regularity problem:

Lemma 2 (i) For $\tilde{F}<1, h_{0}$ is a $C^{\infty}$ function,

(ii) For $\tilde{F} \geq 1, h_{0}$ is a $C^{\infty}$ function everywhere but at 0 where the jump of derivative is $-2 a(\tilde{F})$ with a $(\tilde{F})$ the first positive solution of the equation

$$
a(\tilde{F})=\tilde{F} \sin (a(\tilde{F}))
$$

(Note that $a$ is an increasing function from $[1,+\infty)$ onto $[0, \pi]$ and $a(\pi / 2)=$ $\pi / 2)$,

(iii) In any case $h_{0}$ is decreasing on the interval $[0, \pi]$. Setting $V(\tilde{F}):=h(0)-$ $h(\pi)$, we have:

$$
V(\tilde{F})= \begin{cases}2 \tilde{F} & \text { if } \tilde{F}<1 \\ a(\tilde{F})^{2} / 2+\tilde{F}+\sqrt{\tilde{F}^{2}-(a(\tilde{F}))^{2}} & \text { if } 1<\tilde{F}<\pi / 2 \\ a(\tilde{F})^{2} / 2+\tilde{F}-\sqrt{\tilde{F}^{2}-(a(\tilde{F}))^{2}} & \text { if } \pi / 2<\tilde{F}\end{cases}
$$

and

$$
h_{0}^{\prime}(z)=-\sqrt{2} \sqrt{\tilde{F}-h_{0}(z)} \frac{\sqrt{\tilde{F}+1-\sqrt{1+\tilde{F}^{2}-2 h_{0}(z)}}}{\sqrt{\left.\tilde{1}-F+\sqrt{1+\tilde{F}^{2}-2 h_{0}(z)}\right)}} .
$$

This lemma is illustrated in Fig. 4. 
Proof Let us start by preliminary remarks. The problem

$$
\max _{\theta}\left(z \theta-G_{0}(\theta)\right)
$$

admits at least one solution $\theta(z)$ which has to satisfy $z=G_{0}^{\prime}(\theta(z))$ that is

$$
z=\theta(z)-\tilde{F} \sin (\theta(z))
$$

When this solution is unique and when $G_{0}^{\prime \prime}(\theta(z))>0$, local inversion Theorem states that $\theta(z)$ is an increasing $C^{\infty}$ function. Therefore $G_{0}^{*}(z)=z \theta(z)-$ $G_{0}(\theta(z))$ and consequently $h_{0}$ are of class $C^{\infty}$. In that case we have

$$
\begin{aligned}
\left(G_{0}^{*}\right)^{\prime}(z) & =\theta(z)+\left(z-G_{0}^{\prime}(\theta(z))\right) \theta^{\prime}(z) \\
& =\theta(z)+(z-\theta(z)+\tilde{F} \sin (\theta(z))) \theta^{\prime}(z)=\theta(z)
\end{aligned}
$$

and so

$$
h_{0}^{\prime}(z)=z-\left(G_{0}^{*}\right)^{\prime}(z)=z-\theta(z)=-F \sin (\theta(z)) \leq 0 .
$$

We also have

$$
h_{0}(z)=\frac{z^{2}}{2}-\left(G_{0}^{*}\right)^{\prime}(z)=\frac{(z-\theta(z))^{2}}{2}-\tilde{F} \cos (\theta(z))
$$

and, using (31),

$$
h_{0}(z)=\frac{\tilde{F}^{2}\left(1-\cos ^{2}(\theta(z))\right)}{2}+\tilde{F} \cos (\theta(z))
$$

Therefore $^{3}$

$$
\tilde{F} \cos (\theta(z))=1-\sqrt{1+\tilde{F}^{2}-2 h_{0}(z)}
$$

and so $^{4}$

$$
\begin{aligned}
h_{0}^{\prime}(z) & =-\sqrt{\tilde{F}^{2}-\left(1-\sqrt{\left.1+\tilde{F}^{2}-2 h_{0}(z)\right)^{2}}\right.} \\
& =-\sqrt{2} \sqrt{\tilde{F}-h_{0}(z)} \frac{\sqrt{\tilde{F}+1-\sqrt{1+\tilde{F}^{2}-2 h_{0}(z)}}}{\sqrt{\left.\tilde{1}-F+\sqrt{1+\tilde{F}^{2}-2 h_{0}(z)}\right)}} .
\end{aligned}
$$

3 The justification of the minus sign before the square root involves some cumbersome computations.

4

$$
\begin{gathered}
h_{0}^{\prime}(z)=-\sqrt{\left.\tilde{F}-1+\sqrt{1+\tilde{F}^{2}-2 h_{0}(z)}\right)} \sqrt{\tilde{F}+1-\sqrt{1+\tilde{F}^{2}-2 h_{0}(z)}} . \\
h_{0}^{\prime}(z)=-\frac{\left.\sqrt{(\tilde{F}-1)^{2}-\left(1+\tilde{F}^{2}-2 h_{0}(z)\right.}\right)}{\sqrt{\left.\tilde{F}-1-\sqrt{1+\tilde{F}^{2}-2 h_{0}(z)}\right)}} \sqrt{\tilde{F}+1-\sqrt{1+\tilde{F}^{2}-2 h_{0}(z)}} . \\
h_{0}^{\prime}(z)=-\sqrt{2} \sqrt{-h_{0}(z)+\tilde{F}} \frac{\sqrt{\tilde{F}+1-\sqrt{1+\tilde{F}^{2}-2 h_{0}(z)}}}{\sqrt{\left.\tilde{-} F+1+\sqrt{1+\tilde{F}^{2}-2 h_{0}(z)}\right)}} .
\end{gathered}
$$


When $\tilde{F}<1$, previous remarks apply because the function $G_{0}$ is a strictly convex $C^{\infty}$ function. Point (i) and point (iii) for the case $\tilde{F}<1$ come directly: we only have to focus on the case $\tilde{F} \geq 1$.

Let us now prove that the restriction of $G$ on the interval $(a(\tilde{F}), \pi)$ is strictly convex. As $G^{\prime \prime}(\theta):=1-\tilde{F} \cos (\theta)$ is strictly increasing, it is enough to check that $G^{\prime \prime}(a(\tilde{F})) \geq 0$. This is trivial if $\tilde{F} \geq \pi / 2$ because $a(\tilde{F}) \in(\pi / 2, \pi)$ and so $\cos (a(\tilde{F}))<0$. This is a bit more complicated if $1 \leq \tilde{F} \leq \pi / 2$ : $a(\tilde{F}) \in(\pi / 2, \pi)$ and we can write

$$
G^{\prime \prime}(a(\tilde{F}))=1-\tilde{F} \cos (a(\tilde{F}))=1-\sqrt{\tilde{F}^{2}-(a(\tilde{F}))^{2}}
$$

The results follows ${ }^{5}$ from the properties of the equation (28).

We notice now that, by definition of $a(\tilde{F})$,

$$
G(\theta) \geq 0 \times \theta+G(a(\tilde{F})),
$$

for any $\theta$. On the other hand $T(\theta):=\pi(\theta-\pi)+G(\pi)$ is tangent to $G$ at $\theta=\pi$ and satisfies, for any $\theta \neq \pi$,

$$
G(\theta)-T(\theta)=\frac{1}{2}(\theta-\pi)^{2}+\tilde{F}(\cos (\theta)+1)>0 .
$$

As a consequence, owing to strict convexity, the problem (30) admits, for any $z \in(0, \pi]$, a unique solution $\theta(z)$. This solution belongs to $(a(\tilde{F}), \pi]$ and the preliminary remarks apply. The function $h_{0}$ is of class $C^{\infty}$ on $(0, \pi]$ and, as $\left(G^{*}\right)^{\prime}\left(0^{+}\right)=a(\tilde{F})$, we get the right derivative $h_{0}^{\prime}\left(0^{+}\right)=-a(\tilde{F})$. By symmetry, $h_{0}$ is also of class $C^{\infty}$ on the interval $[-\pi, 0)$ and therefore the function $h_{0}$ admits a unique singularity at $z=0$ on the periodicity interval $[-\pi, \pi]$ with a jump of derivative $-2 a(\tilde{F})$ at $z=0 .{ }^{6}$ Point (ii) is proven.

${ }^{5}$ In order to prove that $G^{\prime \prime}(a(\tilde{F}))>0$ we have to prove that $a(\tilde{F})>\sqrt{\tilde{F}^{2}-1}$. Owing to the properties of equation (28), it is enough to check that $\sqrt{\tilde{F}^{2}-1}<\tilde{F} \sin \left(\sqrt{\tilde{F}^{2}-1}\right)$ or equivalently that, for any $\alpha \in\left(0, \sqrt{\pi^{2} / 4-1}\right)$,

$$
\sin (\alpha)-\frac{\alpha}{\sqrt{1+\alpha^{2}}}>0
$$

We first notice that, on the interval $(0, \pi)$ owing to Taylor-Lagrange expansion, $\sin (\alpha) \geq$ $\alpha-\frac{\alpha^{3}}{6}$ and then we are reduced to study the inequality

$$
\alpha-\frac{\alpha^{3}}{6}>\frac{\alpha}{\sqrt{1+\alpha^{2}}}
$$

or equivalently

or

$$
1+\frac{\alpha^{4}}{36}-\frac{\alpha^{2}}{3}>\frac{1}{1+\alpha^{2}}
$$

$$
0<24-11 \alpha^{2}+\alpha^{4} .
$$

The result is ensured by the fact that this inequality is true for any $\alpha^{2} \in(0,3)$.

${ }^{6}$ It may also be usefull to remark that, as

$$
\theta^{\prime}(z)=1 / G^{\prime \prime}(\theta(z))=1 /(1-\tilde{F} \cos (\theta(z))) \geq 0,
$$



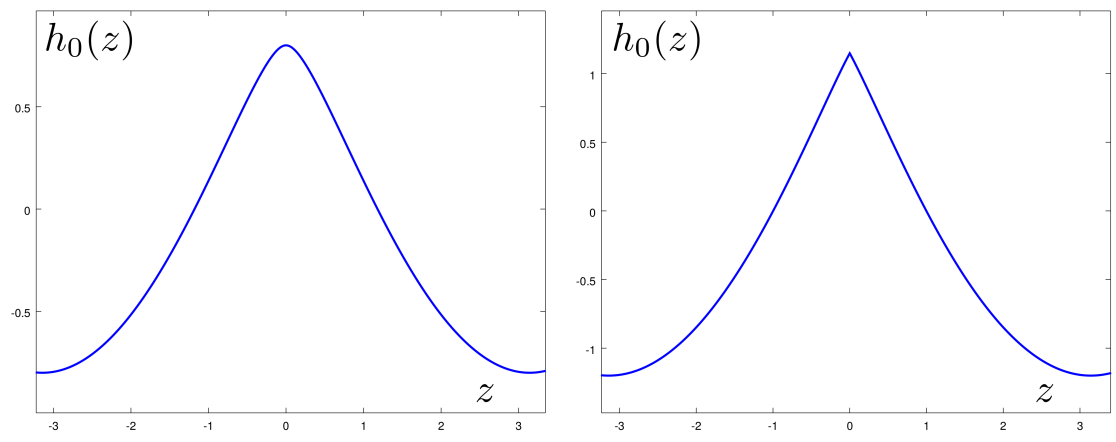

Fig. 4 Plot of the function $h_{0}$ in case of $\tilde{F}=0.8$ (left) and $\tilde{F}=1.2$ (right). It can be seen that the derivative jumps at $s=0$ in the second case.

It remains to compute the variation of $h_{0}$ in the case $\tilde{F} \geq 1$. We have $\theta(0)=a(\tilde{F})$ and $\theta(\pi)=\pi$. From $(34)$, we directly deduce

$$
V(\tilde{F}):=h(0)-h(\pi)=a(\tilde{F})^{2} / 2+\tilde{F}(\cos (a(\tilde{F}))+1)
$$

and we get the desired result by taking into account the $\operatorname{sign}$ of $\cos (a(\tilde{F}))$.

\subsubsection{Buckling load}

When $\gamma=0$, the constant function $\phi=0$ is a critical point of Problem (18). When $F$ is small enough, this trivial solution is the minimizer of the energy but when $F$ increases, other critical solutions may exist. This phenomenon is known as the buckling of the beam under compressive load. We determine in this section the value of the load above which several critical solutions exist.

Lemma 3 The number of critical solutions of the problem

$$
\min _{\phi}\left\{\int_{0}^{1}\left(\frac{1}{2}\left(\phi^{\prime}(s)\right)^{2}+\tilde{K}_{2} h_{0}(\phi(s))\right) d s ; \phi(0)=0\right\}
$$

with

$$
h_{0}(z)=\frac{(z-\theta(z))^{2}}{2}+\tilde{F} \cos (\theta(z))
$$

and

$$
z=\theta(z)-\tilde{F} \sin (\theta(z))
$$

is larger than one if and only if $\tilde{F}>\frac{1}{1+\frac{4 \tilde{K}_{2}}{\pi^{2}}}$.

we have

$$
h^{\prime \prime}(z)=1-1 /(1-\tilde{F} \cos (\theta(z)))=-\tilde{F} \cos (\theta(z)) /(1-\tilde{F} \cos (\theta(z))) .
$$

Hence $h^{\prime \prime}(z)$ has the opposite sign to $\cos (\theta(z))$. It remains positive on the interval $(a(\tilde{F}), \pi)$ if and only if $a(\tilde{F}) \geq \pi / 2$, in otherwords, if $\tilde{F} \geq \pi / 2$. In that case $h$ is convex between two successive singularities. If $1<\tilde{F}<\pi / 2$ there exists an inflexion point and $h$ is concave in a vicinity of the singularities. 
Proof It is easy to check that critical solutions of (37) are the solutions, for some constant $C$, of the differential equation

$$
\frac{1}{2}\left(\phi^{\prime}(s)\right)^{2}-\tilde{K}_{2} h_{0}(\phi(z))=C
$$

with the boundary conditions $\phi(0)=0$ and $\phi^{\prime}(1)=0$.

Let us set $C=\tilde{K}_{2}\left(\frac{k^{2}}{2}-h_{0}(0)\right)$ or equivalently $\left|\phi^{\prime}(0)\right|=k \sqrt{\tilde{K}_{2}}$ and let us study for which values of $k$ the solution of (40) with initial conditions $\phi(0)=0$, $\phi^{\prime}(0)=k \sqrt{\tilde{K}_{2}}$ satisfies $\phi^{\prime}(1)=0$. If $k^{2} / 2>V(F)=h_{0}(0)-h_{0}(\pi)$ it is clearly impossible.

We let apart the trivial solution $k=0, \phi=0$ and by symmetry we focus on the case $\phi^{\prime}(0)=k \sqrt{\tilde{K}_{2}}>0$. We call $\phi_{k}$ the solution of (40) and we denote $L(k)$ the smallest positive value of $s$ such that $\phi_{k}^{\prime}(s)=0$. We also denote $z_{k}=\phi_{k}(L(k))$ which implies

$$
h_{0}\left(z_{k}\right)=h_{0}(0)-\frac{k^{2}}{2} .
$$

As $\phi_{k}$ is monotonous on $[0, L(k)]$ we can write

$$
L(k)=\int_{0}^{z_{k}}\left(\left(\phi_{k}\right)^{(-1)}\right)^{\prime}(z) d z=\frac{1}{\sqrt{\tilde{K}_{2}}} \int_{0}^{z_{k}} \frac{1}{\sqrt{k^{2}+2\left(h_{0}(z)-h_{0}(0)\right)}} d z
$$

Let us first remark that $L$ is a continuous (not necessarily monotonous) function of $k$ which tends to infinity when $k$ tends to $\sqrt{2\left(h_{0}(0)-h_{0}(\pi)\right)}$. Indeed the integral becomes singular in the vicinity of $\pi$. Things are less clear when $k$ tends to zero. Using the change of variable

$$
v(z):=\arcsin \left(\frac{\sqrt{2\left(h_{0}(0)-h_{0}(z)\right)}}{k}\right),
$$

we get

$$
\sqrt{\tilde{K}_{2}} L(k)=\int_{0}^{\frac{\pi}{2}} j(k \sin (v)) d v
$$

with

$$
j(u):=-\frac{u}{h_{0}^{\prime}\left(h_{0}^{(-1)}\left(h_{0}(0)-\frac{u^{2}}{2}\right)\right)}=-\frac{u}{h_{0}^{\prime}\left(z_{u}\right)}
$$

which becomes, using expression of $h_{0}^{\prime}$ given by Lemma 2 and equation (41),

$$
\begin{aligned}
j(u) & =\frac{u}{\sqrt{2 \tilde{F}-2 h_{0}\left(z_{u}\right)}} \sqrt{\frac{1-\tilde{F}+\sqrt{1+\tilde{F}^{2}-2 h_{0}\left(z_{u}\right)}}{\tilde{F}+1-\sqrt{1+\tilde{F}^{2}-2 h_{0}\left(z_{u}\right)}}} \\
& =\frac{u}{\sqrt{2\left(\tilde{F}-h_{0}(0)\right)+u^{2}}} \sqrt{\frac{1-\tilde{F}+\sqrt{1+\tilde{F}^{2}-2 h_{0}(0)+u^{2}}}{\tilde{F}+1-\sqrt{1+\tilde{F}^{2}-2 h_{0}(0)+u^{2}}}}
\end{aligned}
$$


When $\tilde{F}>1$, one can easily check that $h_{0}(0)<\tilde{F}$. The function $j$ has no singularity, $j(0)=0$ and we can pass to the limit in $(42)$. We obtain $L\left(0^{+}\right)=0$. The theorem of intermediate values ensures the existence of a $k$ in $(0,+\infty)$ such that $L(k)=1$;

When $F \leq 1$, we have $h_{0}(0)=\tilde{F}$ and the expression of $j$ can be simplified

$$
j(u)=\sqrt{\frac{1-\tilde{F}+\sqrt{(1-\tilde{F})^{2}+u^{2}}}{\tilde{F}+1-\sqrt{(1-\tilde{F})^{2}+u^{2}}}}
$$

We see now that $j$ is strictly increasing : $L$ takes values in $(L(0),+\infty)$ and a solution such that $L(k)=1$ exists if and only if

$$
1>\frac{1}{\sqrt{\tilde{K}_{2}}} L(0)=\frac{1}{\sqrt{\tilde{K}_{2}}} \int_{0}^{\frac{\pi}{2}} j(0) d v=\frac{1}{\sqrt{\tilde{K}_{2}}} \frac{\pi}{2} \sqrt{\frac{1-\tilde{F}}{\tilde{F}}}
$$

or equivalently

$$
\tilde{F}>\tilde{F}_{c}:=\frac{1}{1+\frac{4 \tilde{K}_{2}}{\pi^{2}}} .
$$

It is interesting to write this critical load in terms of the original parameters. It becomes

$$
F \geq F_{c} \frac{1}{1+\frac{F_{c}}{K_{2}}} \quad \text { with } \quad F_{c}:=\frac{\pi^{2} K_{1}}{4 L^{2}}
$$

The quantity $F_{c}$ is known to be the critical load for a clamped-free Euler beam (the historical reference is [1]; for interesting recent developments see also [34-38]). The previous formula provides the correction to be applied for a Timoshenko beam. As expected the critical value tends to $F_{c}$ when the Timoshenko coupling parameter $K_{2}$ is very large.

Note also that the number of critical solutions increases with $\tilde{F}$. It passes from 1 to 3 when $\tilde{F}$ reaches the critical value $\tilde{F}_{c}$. It can be shown that more generally, it passes from $2 n-1$ to $2 n+1$ when $\tilde{F}$ reaches the value

$$
\frac{1}{1+\frac{4(2 n-1)^{2} \tilde{K}_{2}}{\pi^{2}}}
$$

To our knowledge, this is the first estimate for the critical load of a beam with energy model (8). 


\subsubsection{Euler-Lagrange equations}

A formal computation starting from (18) leads to the following Euler Lagrange equations for an inextensible Timoshenko beam under the end load $\boldsymbol{F}=F_{1} \boldsymbol{e}_{1}+$ $F_{2} e_{2}:$

$$
\left\{\begin{array}{l}
\phi^{\prime \prime}(s)=\frac{K_{2}}{K_{1}}(\phi(s)-\theta(s)) \\
\phi(s)=\theta(s)-\frac{F_{1}}{K_{2}} \sin (\theta(s))+\frac{F_{2}}{K_{2}} \cos (\theta(s)) \\
\phi(0)=\phi^{\prime}(1)=0
\end{array}\right.
$$

Substituting $\phi$ in the first equation we obtain the boundary value problem for $\theta(s)$ :

$$
\left\{\begin{array}{l}
\theta^{\prime \prime}(s)=\frac{\left(F_{2} \cos \theta-F_{1} \sin \theta\right)\left(K_{2}+K_{1} \theta^{\prime 2}\right)}{K_{1}\left(K_{2}-F_{1} \cos \theta-F_{2} \sin \theta\right)} \\
\theta(0)=-\frac{F_{1}}{K_{2}} \sin (\theta(0))+\frac{F_{2}}{K_{2}} \cos (\theta(0)) \\
\theta^{\prime}(1)=0
\end{array}\right.
$$

Of course a minimizer of the problem (18) will not have, in general, enough regularity to solve (43) in a strong sense (see noticeable examples in [39]). A stationary pair $(\phi, \theta)$ solves the integral equation:

$$
\phi(s)=\int_{0}^{s} d t \int_{t}^{1} \frac{K_{2}}{K_{1}}(\phi-\theta) d x
$$

Recalling that $\phi$ is in $H^{1}$ (i.e. it has a continuous representative), in the intervals on which $\theta$ is $C^{\infty}$ the previous integral representation implies $C^{2}$ regularity for $\phi$, and applying the argument recursively one obtains $C^{\infty}$ regularity for both $\phi$ and $\theta$ almost everywhere, i.e. except at the points $s$ such that $G^{*}(\phi(s))$ is not differentiable. We already showed that, in case $\tilde{F}<1, \theta$ solves the equations (44) in the whole interval $[0,1]$.

\subsection{Regularized Timoshenko model}

Since the integrand in (16) is convex and coercive with respect to the highest order derivatives, by a well-known result of calculus of variations (see [29]), the problem:

$$
\left\{\begin{array}{l}
\min E(\phi, \theta):=\int_{0}^{1}\left[\frac{K_{1}}{2} \phi^{2}+\frac{K_{2}}{2}(\phi-\theta)^{2}+\frac{K_{3}}{2} \theta^{2}+F_{1} \cos \theta+F_{2} \sin \theta\right] d s \\
\theta \in H^{1}[0,1], \quad \phi \in H^{1}[0,1], \quad \theta(0)=\phi(0)=0
\end{array}\right.
$$

admits at least one solution, say $(\bar{\phi}, \bar{\theta})$. Moreover, the minimizer $(\bar{\phi}, \bar{\theta})$ verifies in a weak form the relative Euler-Lagrange equations: 


$$
\left\{\begin{array}{l}
\phi^{\prime \prime}=\frac{K_{2}}{K_{1}}(\phi-\theta) \\
\theta^{\prime \prime}=\frac{K_{2}}{K_{3}}(\theta-\phi)-\frac{F_{1}}{K_{3}} \sin \theta+\frac{F_{2}}{K_{3}} \cos \theta
\end{array}\right.
$$

which means that a minimizing pair $(\bar{\phi}, \bar{\theta})$ is a fixed point for the following nonlinear integral operator:

$$
\begin{aligned}
& (\phi, \theta) \longmapsto \mathbb{T}(\phi, \theta):=\left(\mathbb{T}_{1}(\phi, \theta), \mathbb{T}_{2}(\phi, \theta)\right) \\
& \mathbb{T}_{1}(\phi, \theta):=\frac{K_{2}}{K_{1}} \int_{0}^{s} d x \int_{1}^{x} d t[\phi(t)-\theta(t)] \\
& \mathbb{T}_{2}(\phi, \theta):=\frac{1}{K_{3}} \int_{0}^{s} d x \int_{1}^{x} d t\left[K_{2}(\theta(t)-\phi(t))+F_{1} \sin \theta-F_{2} \cos \theta\right]
\end{aligned}
$$

Clearly $\mathbb{T}(\phi, \theta)$ is in $C^{2} \times C^{2}$ if $(\phi, \theta) \in H^{1} \times H^{1}$. Therefore, $(\bar{\phi}, \bar{\theta})$ verify (47) in a strong (pointwise) sense. Finally, since $(\bar{\phi}, \bar{\theta})$ is a fixed point for $\mathbb{T}$, the previous argument can be applied recursively, which implies $C^{\infty}$ regularity for the minimizer.

\section{Numerical simulations}

\subsection{Results on the absolute minimizer}

In this section we want to show some numerical results on the absolute minimizers of energy models (18) and (46). The main aim of these numerical investigations is to show the effect of the introduction of the regularizing term in $\theta^{\prime 2}$ in the deformation energy, and indeed we will "perturb" the problem (18) by means of a term of the type $\varepsilon \theta^{\prime 2}$ with $\varepsilon \ll 1$.

The numerical procedure to find the minimizer is different in the two cases of models (18) and (46). In the first case, we used a direct numerical minimization (based on finite elements) to find a solution of the problem $P(18)$. We searched $\tilde{\theta}$ and $\tilde{\phi}$ respectively in the set of piecewise constant and piecewise linear functions (which are dense respectively in $L^{2}$ and $H^{1}$ ). As an internal consistency test, we also checked that the solution $\tilde{\theta}$ coincides with the one obtained from $\tilde{\phi}$ by means of (20).

On the other hand, in the case of the problem (46), the regularity proven in section 3.2 allowed us to use the Euler-Lagrange equations. The corresponding boundary value problem was solved by means of a two-dimensional shooting technique. Specifically, an explicit Euler method (with an integration step $\eta=10^{-3}$ ) has been implemented to solve a set of initial value problems parameterized by means of two parameters:

$$
\left\{\begin{array}{l}
\phi^{\prime \prime}=\frac{K_{2}}{K_{1}}(\phi-\theta) \quad \theta^{\prime \prime}=\frac{K_{2}}{K_{3}}(\theta-\phi)-\frac{F_{1}}{K_{3}} \sin \theta+\frac{F_{2}}{K_{3}} \cos \theta \\
\phi(0)=0 \quad \theta(0)=0 \\
\phi^{\prime}(0)=A_{1} \quad \theta^{\prime}(0)=A_{2}
\end{array}\right.
$$


We studied the behavior of the vector of boundary values $\boldsymbol{p}:=\left(\phi^{\prime}(1), \theta^{\prime}(1)\right)$ as a function of $A_{1}$ and $A_{2}$, and employed a direct minimizer to find the points in the space of parameters where $\|\boldsymbol{p}\|$ is minimal. More precisely, we introduced a square grid (with step length $\ell \approx 10^{-3}$ ) on the plane $\left(A_{1}, A_{2}\right)$ and searched for the cells of the grid in which the local minima of $\|\boldsymbol{p}\|$ are situated.

In the following graphs we show some relevant results obtained with these two procedures. The parameters employed in the various cases are indicated in the captions.
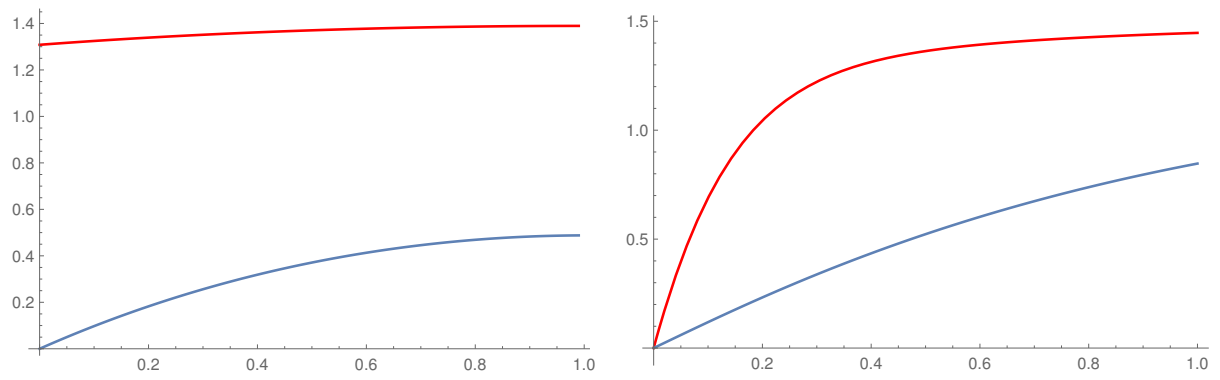

Fig. 5 The minimizing pair $(\tilde{\phi}, \tilde{\theta})$ in case of transverse end load for a nonlinear Timoshenko energy model (left) and regularized model obtained adding to the previous a term in $\theta^{\prime 2}$ with a small coefficient (right). The parameters are $F_{1}=0, F_{2}=10, K_{1} / 2=1, K_{2} / 2=1$ (left) and the same with $K_{3} / 2=0.1$ (right). Notice that on the left we have $\tilde{\theta}(0) \neq 0$.
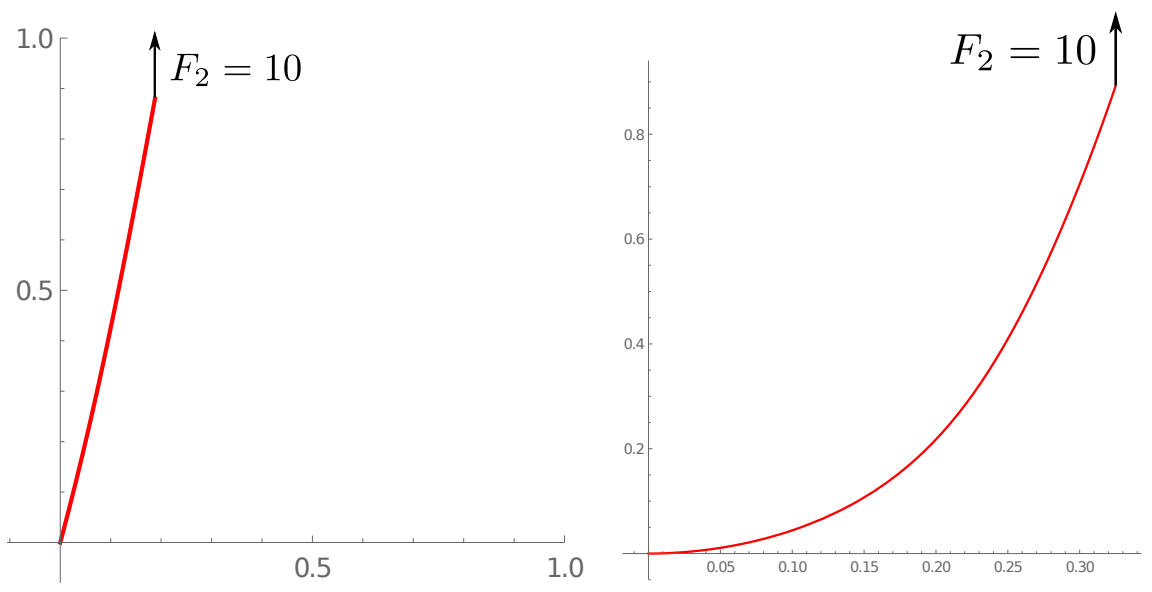

Fig. 6 The deformed shape of the beam corresponding to the case of transverse end load for a nonlinear Timoshenko energy model (left) and regularized model obtained adding to the previous a term in $\theta^{\prime 2}$ with a small coefficient (right). The parameters are the same as in the previous figure. 

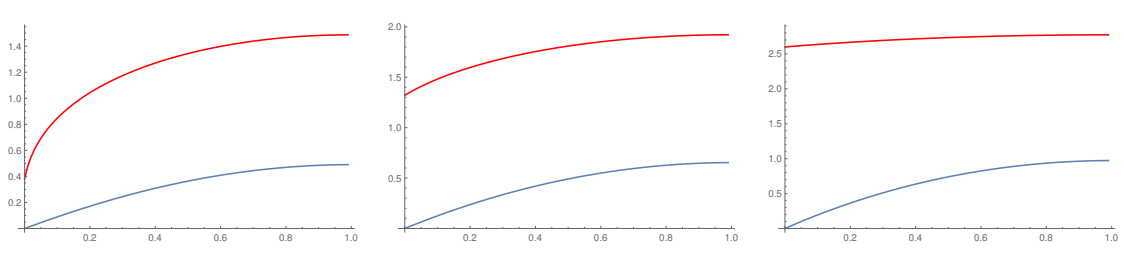

Fig. 7 The minimizing pair $(\tilde{\phi}, \tilde{\theta})$ corresponding to three different compressive end loads for a nonlinear Timoshenko energy model. The parameters are $F_{2}=0, K_{1} / 2=1, K_{2} / 2=1$ in the three plots, while $F_{1}=2$ (left), $F_{1}=2.7$ (center) and $F_{1}=10$ (right). Notice that the absolute value of $\tilde{\theta}(0)$ increases with $F_{1}$.
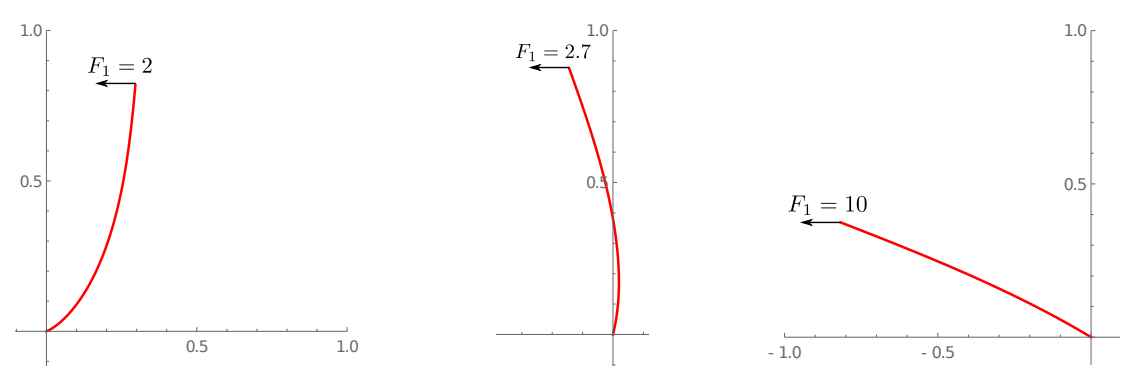

Fig. 8 The deformed shape of the beam corresponding to three different compressive end loads for a nonlinear Timoshenko energy model. The parameters are $F_{2}=0, K_{1} / 2=$ $1, K_{2} / 2=1$ in the three plots, while $F_{1}=2$ (left), $F_{1}=2.7$ (center) and $F_{1}=10$ (right).

4.2 Results on other stationary points

In this section we want to show some curled equilibria of the nonlinear Timoshenko beam model (8) and of the regularized model (16), which resemble the ones presented for an inextensible Euler beam (in case of distributed load) in [30]. In doing so we assume that stationary points different from the absolute minimizer solve Euler-Lagrange equations (43).

Solving the boundary value problem by means of a shooting technique (similarly to what explained in the previous section), we found the curled solutions shown in Fig. 4.2. It is interesting to notice that, passing from the simple Euler model to nonlinear Timoshenko and regularized Timoshenko, the property of having such kind of curled solutions is still kept. The complete study of local minima of the nonlinear Timoshenko beam model is not trivial and would probably require ideas from the theory of phase transitions (see $[41,42]$ ). 

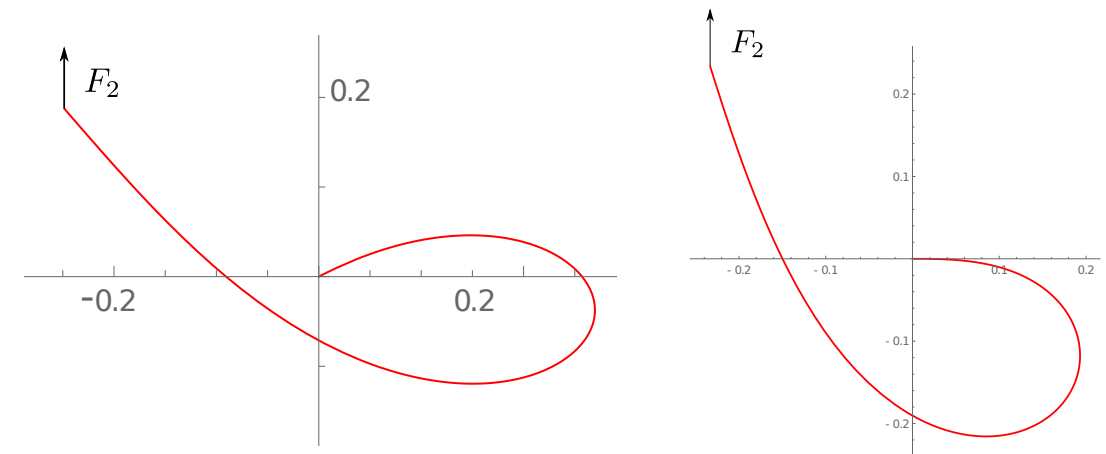

Fig. 9 A curled equilibrium shape for nonlinear Timoshenko model (left); the parameters are: $K_{1} / 2=1, K_{2} / 2=20, F_{2}=25$. The same kind of equilibrium shape for a regularized Timoshenko model (right); the parameters are: $K_{1} / 2=1, K_{2} / 2=1, K_{3} / 2=0.1, F_{2}=10$.

\section{Conclusions}

The importance of geometrical nonlinearities is increasingly important in modern structural mechanics (see e.g. [40]) and in general in elasticity theory (see [43-48]). On the other hand, beam theory, and especially generalized beam theory, is particularly interesting nowadays in view of applications to lattice systems $[49,50,31]$, and in this context pantographic structures are naturally leading to the problem of large deformations of the fibers (see for instance [51-57]).

This paper dealt with the problem of geometrically nonlinear deformation of generalized Timoshenko beam models, obtained by means of a formal homogenization starting from a microstructured 1D system. We considered both a straightforward generalization of the customary linearized model (7) and the model obtained introducing in the microstructure an additional rotational spring entailing a term in $\theta^{\prime 2}$ in the deformation energy density. We proved well-posedness of the variational problem concerning a clamped beam with generic end load, as well as some properties of the minimizers, and presented and discussed some numerical simulations.

The main open problem connected with the content of the work is the generalization of the results to the case of a distributed load, which would allow the use of the result in the modeling of lattice fibrous systems with beams connected each other with finely spaced pivots $([32,33,51])$. However, this generalization is not trivial as it leads to a non-autonomous variational problem. We remark indeed that the given demonstrations do not generalize trivially to the case of a distributed load, as the monotonicity properties of the minimizer discussed in [28] are not available in general.

Finally, we want to mention that the nonlinear differential equations describing the nonlinear model introduced here are suitable to account for many different phenomena. For instance, they can describe the motion of a pendulum consisting of a weight linked by means of a Hooke's spring to a charge of negligible 
mass oscillating in an electromagnetic field (see [58]); in case of distributed load the electromagnetic field would be time-dependent. Therefore, the interest of studying the nonlinear energy model also in case of distributed load goes probably well beyond 1D elasticity.

\section{Compliance with Ethical Standards}

The authors declare that they have no conflict of interest.

\section{References}

1. Leonhard Euler. Methodus inveniendi lineas curvas maximi minimive proprietate gaudentes, sive solutio problematis isoperimetrici lattissimo sensu accepti, chapter Additamentum 1. eulerarchive.org E065, 1744.

2. Bernoulli, D.. The 26th letter to Euler. In Correspondence Mathmatique et Physique, volume 2. P. H. Fuss, October 1742.

3. Bernoulli, J. Quadratura curvae, e cujus evolutione describitur inflexae laminae curvatura. Die Werke von Jakob Bernoulli, 223-227.

4. Lagrange, J. L. Mécanique analytique (Vol. 1-2), Mallet-Bachelier, 1853.

5. Mora, M. G., \& Mller, S. (2004). A nonlinear model for inextensible rods as a low energy $\Gamma$-limit of three-dimensional nonlinear elasticity. In Annales de l'IHP Analyse non linaire (Vol. 21, No. 3, pp. 271-293).

6. Pideri, C., \& Seppecher, P. (2006). Asymptotics of a non-planar rod in non-linear elasticity. Asymptotic Analysis, 48(1, 2), 33-54.

7. Eugster, S. R. (2015). Geometric continuum mechanics and induced beam theories (Vol. 75). New York: Springer.

8. Eugster, S., \& Glocker, C. (2014). Determination of the Transverse Shear Stress in an Euler-Bernoulli Beam Using Non-Admissible Virtual Displacements. PAMM, 14(1), 187188.

9. Timoshenko, S.P. (1921). On the correction factor for shear of the differential equation for transverse vibrations of prismatic bar. Philosophical Magazine, 6(41), 744.

10. Plantema, F.J. Sandwich construction; the bending and buckling of sandwich beams, plates, and shells. 1966

11. Turco, E., Barcz, K., Pawlikowski, M., \& Rizzi, N. L. (2016). Non-standard coupled extensional and bending bias tests for planar pantographic lattices. Part I: numerical simulations. Zeitschrift fr angewandte Mathematik und Physik, 67(5), 122.

12. Birsan, M., Altenbach, H., Sadowski, T., Eremeyev, V. A., \& Pietras, D. (2012). Deformation analysis of functionally graded beams by the direct approach. Composites Part B: Engineering, 43(3), 1315-1328.

13. Eugster, S. R. (2015). Augmented Nonlinear Beam Theories. In Geometric Continuum Mechanics and Induced Beam Theories (pp. 101-115). Springer International Publishing.

14. Piccardo, G., Ferrarotti, A., \& Luongo, A. (2016). Nonlinear Generalized Beam Theory for open thin-walled members. Mathematics and Mechanics of Solids, 1081286516649990.

15. Luongo, A., \& Zulli, D. (2013). Mathematical models of beams and cables. John Wiley \& Sons.

16. Ruta, G. C., Varano, V., Pignataro, M., \& Rizzi, N. L. (2008). A beam model for the flexural-torsional buckling of thin-walled members with some applications. Thin-Walled Structures, 46(7), 816-822.

17. Hamdouni, A., \& Millet, O. (2006). An asymptotic non-linear model for thin-walled rods with strongly curved open cross-section. International Journal of Non-Linear Mechanics, 41(3), 396-416.

18. Grillet, L., Hamdouni, A., \& Millet, O. (2004). An asymptotic non-linear model for thin-walled rods. Comptes Rendus Mcanique, 332(2), 123-128. 
19. Grillet, L., Hamdouni, A., \& Millet, O. (2005). Justification of the kinematic assumptions for thin-walled rods with shallow profile. Comptes Rendus Mcanique, 333(6), 493-498.

20. Hamdouni, A., \& Millet, O. (2011). An asymptotic linear thin-walled rod model coupling twist and bending. International Applied Mechanics, 46(9), 1072-1092.

21. dell'Isola, F., Steigmann, D., \& Della Corte, A. (2016). Synthesis of fibrous complex structures: designing microstructure to deliver targeted macroscale response. Applied Mechanics Reviews, 67(6).

22. Eugne et Franois Cosserat, Thorie des corps dformables. Paris, Librairie Scientifique A. Hermann et Fils, 1909

23. Forest, S. (2005). Mechanics of Cosserat media - an introduction. Ecole des Mines de Paris, Paris, 1-20.

24. Altenbach, J., Altenbach, H., \& Eremeyev, V. A. (2010). On generalized Cosserattype theories of plates and shells: a short review and bibliography. Archive of Applied Mechanics, 80(1), 73-92.

25. Pietraszkiewicz, W., \& Eremeyev, V. A. (2009). On vectorially parameterized natural strain measures of the non-linear Cosserat continuum. International Journal of Solids and Structures, 46(11), 2477-2480.

26. Kannan, R., and Krueger, C. K. (2012). Advanced analysis: on the real line. Springer Science \& Business Media.

27. Rockafellar, R. T. (2015). Convex analysis. Princeton university press.

28. Cupini, G., Guidorzi, M., and Marcelli, C. (2007). Necessary conditions and nonexistence results for autonomous nonconvex variational problems. Journal of Differential Equations, 243(2), 329-348.

29. Fonseca, I., \& Leoni, G. (2007). Modern Methods in the Calculus of Variations: $L^{p}$ Spaces. Springer Science \& Business Media.

30. Della Corte, A., dell'Isola, F., Esposito, R., and Pulvirenti, M. (2016). Equilibria of a clamped Euler beam (Elastica) with distributed load: large deformations. M3AS (2017), doi: http://dx.doi.org/10.1142/S0218202517500221

31. Pipkin, A. C. (1980). Some developments in the theory of inextensible networks. Quarterly of Applied Mathematics, 38(3), 343-355.

32. Steigmann, D. J., \& Pipkin, A. C. (1991). Equilibrium of elastic nets. Philosophical Transactions of the Royal Society of London A: Mathematical, Physical and Engineering Sciences, 335(1639), 419-454

33. dell'Isola, F., Giorgio, I., Pawlikowski, M., \& Rizzi, N. L. (2016, January). Large deformations of planar extensible beams and pantographic lattices: heuristic homogenization, experimental and numerical examples of equilibrium. In Proc. R. Soc. A (Vol. 472, No. 2185, p. 20150790). The Royal Society.

34. Ferretti, M., D'Annibale, F., Luongo, A. (2017). Flexural-torsional flutter and buckling of braced foil beams under a follower force, Mathematical Problems in Engineering, in press.

35. Luongo, A., D'Annibale, F. (2013). Double zero bifurcation of non-linear viscoelastic beams under conservative and non-conservative loads, International Journal of Non-Linear Mechanics, 55, 128-139.

36. Luongo, A., D'Annibale, F. (2012). Bifurcation analysis of damped visco-elastic planar beams under simultaneous gravitational and follower forces, International Journal of Modern Physics B, 26(25).

37. Di Egidio, A., Luongo, A., Paolone, A., Linear and nonlinear interactions between static and dynamic bifurcations of damped planar beams, International Journal of Non-Linear Mechanics, 42(1), 88-98, 2007.

38. Goriely, A., Vandiver, R., \& Destrade, M. (2008, November). Nonlinear euler buckling. In Proceedings of the Royal Society of London A: Mathematical, Physical and Engineering Sciences (Vol. 464, No. 2099, pp. 3003-3019). The Royal Society.

39. Ball, J. M., \& Mizel, V. J. (1987). One-dimensional variational problems whose minimizers do not satisfy the Euler-Lagrange equation. In Analysis and Thermomechanics (pp. 285-348). Springer Berlin Heidelberg.

40. Fertis, D. G. (2006). Nonlinear structural engineering. Springer-Verlag Berlin Heidelberg.

41. Lawrie, I. D. (1987). Phase transitions. Contemporary Physics, 28(6), 599-601. 
42. De Masi, A., Presutti, E., \& Tsagkarogiannis, D. (2011). Fourier law, phase transitions and the stationary Stefan problem. Archive for rational mechanics and analysis, 201(2), 681-725.

43. McBride, A.T., Javili, A., Steinmann, P., \& Bargmann, S. (2011). Geometrically nonlinear continuum thermomechanics with surface energies coupled to diffusion. Journal of the Mechanics and Physics of Solids, 59(10), 2116-2133.

44. Eremeyev, V. A., \& Pietraszkiewicz, W. (2004). The nonlinear theory of elastic shells with phase transitions. Journal of Elasticity, 74(1), 67-86.

45. Steigmann, D. J. (2013). Koiter's shell theory from the perspective of three-dimensional nonlinear elasticity. Journal of Elasticity, 111(1), 91-107.

46. Steigmann, D. J. (2009). A concise derivation of membrane theory from threedimensional nonlinear elasticity. Journal of Elasticity, 97(1), 97-101.

47. Forest, S., \& Sievert, R. (2006). Nonlinear microstrain theories. International Journal of Solids and Structures, 43(24), 7224-7245.

48. Ladevèze, P. (2012). Nonlinear computational structural mechanics: new approaches and non-incremental methods of calculation. Springer Science \& Business Media. ISO 690

49. Rivlin, R. S. (1997). Networks of inextensible cords. In Collected Papers of RS Rivlin (pp. 566-579). Springer New York.

50. Pipkin, A. C. (1981). Plane traction problems for inextensible networks. The Quarterly Journal of Mechanics and Applied Mathematics, 34(4), 415-429.

51. Alibert, J. J., Seppecher, P., \& dell'Isola, F. (2003). Truss modular beams with deformation energy depending on higher displacement gradients. Mathematics and Mechanics of Solids, 8(1), 51-73.

52. Scerrato, D., Giorgio, I., \& Rizzi, N. L. (2016). Three-dimensional instabilities of pantographic sheets with parabolic lattices: numerical investigations. Zeitschrift fr angewandte Mathematik und Physik, 67(3), 1-19.

53. Giorgio, I. (2016). Numerical identification procedure between a micro-Cauchy model and a macro-second gradient model for planar pantographic structures. Zeitschrift fr angewandte Mathematik und Physik, 67(4), 95.

54. Turco, E., \& Rizzi, N. L. (2016). Pantographic structures presenting statistically distributed defects: numerical investigations of the effects on deformation fields. Mechanics Research Communications, 77, 65-69.

55. Placidi, L., Andreaus, U., \& Giorgio, I. (2017). Identification of two-dimensional pantographic structure via a linear D4 orthotropic second gradient elastic model. Journal of Engineering Mathematics, 103(1), 1-21.

56. Barchiesi, E., \& Placidi, L. (2017). A Review on Models for the 3D Statics and 2D Dynamics of Pantographic Fabrics. In Wave Dynamics and Composite Mechanics for Microstructured Materials and Metamaterials (pp. 239-258). Springer Singapore.

57. Turco, E., Golaszewski, M., Giorgio, I., \& Placidi, L. (2017). Can a Hencky-Type Model Predict the Mechanical Behaviour of Pantographic Lattices?. In Mathematical Modelling in Solid Mechanics (pp. 285-311). Springer Singapore.

58. Baker, G. L., \& Blackburn, J. A. (2005). The pendulum: a case study in physics. Oxford University Press. 